\title{
A longitudinal study developed to characterize costumers' perceptions on social responsibility
}

\author{
Iudit Bere Semeredi ${ }^{1}$, Cristina Borca $^{2}$, Anca Draghici ${ }^{1}$, Larisa Ivascu ${ }^{1}$, and Dana Fatol ${ }^{1}$ \\ ${ }^{1}$ Politehnica University Timişoara, Faculty of Management in Production and Transportation, 14 \\ Remus str., Timisoara, Romania \\ ${ }^{2}$ Aquatim SA, 11/A Gheorghe Lazar str., Timisoara, Romania
}

\begin{abstract}
The descriptive analysis (based on a longitudinal analysis of the available data from four surveys developed in 2009-2019) aims characterize the customers' perception on social responsibility dimensions in the case of the water company Aquatim of Timisoara, Romania. This is the basis for the communication strategy definition for its efficiency increasing from both perspectives: (1) customers, community, and (2) company. The theoretical and experimental approach characterizes aspects of customers' ecological awareness and satisfaction that have impact on redefining social responsibility policies of the company.
\end{abstract}

\section{Introduction}

Longitudinal studies are a type of survey that primarily uses the method of observation, which entails that they do not involve interfering with the subjects in any means. These studies are also unique in a way that they follow a certain timeline that is entirely dependent on the respondents, which means that data collection could take years depending on the exact timetable put in place [1].

According to the literature, longitudinal analysis methodology is rarely applied in the water sector. Only few studies exist referring to: arsenic exposure from drinking water and risk of premalignant skin lesions [2], residential water use [3], rainfall and coliform contamination [4], the sustainability development of companies [5], but they are based on statistical data available at regional or national level and they do not touch the company (micro) level of investigation.

In the present context study, the longitudinal analysis (based on available data from four surveys developed from 2009 to 2019) aims characterize the customers' perception on social responsibility dimensions in the case of the water company Aquatim of Timisoara, Romania (extended from [6]). For a clear reflection of the studied realities the research presents, in a first stage, the evolution of the dimensions analysed (customers' ecological awareness and satisfaction) in a comparative register for two categories of customers. Second, the trend curves are model to describe the perception on social responsibility dimensions and the aspects of the organizational communication among the company's customers.

* Corresponding author: iudit.bere-semeredi@s,student.upt.ro 


\section{Results and debates of the descriptive analysis}

\subsection{Dimension: Ecological Awareness}

To assess this dimension, responses to questions related to the awareness that poorly treated wastewater produces polluted emissions (rivers) in which it is discharged has been analysed (data presented by [6]). In addition, the treatment costs are included in the structure of service tariffs. Investigating this dimension can provide a first overview of the impact of organizational communication on social responsibility policy (with link to policy, tactics, actions on wastewater treatment) for Aquatim company. In conclusion, the dimension "Ecological Awareness" was described by two variables according to (1):

\section{Environmental awareness $=f($ Wastewater treatment + service payment wastewater treatment plant)

As seen in Figure 1, more than half of respondents in Timisoara stated (in 2009) that "the wastewater that reaches the sewerage network is treated in order not to affect the environment and then evacuated in nature". For the survey conducted in 2015 the question related to this aspect was not included. In 2019, it is noted that the percentage of responses to the social responsibility action on wastewater treatment increased significantly to $79.60 \%$.

The increasing trend associated with the customers' answers to this question reflects the fact that, during the analysis period, organizational communication (strategy, policies, tactics) was better and more suitable for the customers in Timisoara but inadequate, even non-existent for branch customers. Thus, the data analysis presented in Figure 1 pointed out that Timisoara customers have a good perception of the wastewater treatment due to the communication tactics used. This situation is different in the case of customers in the branches, where the communication strategy and policies were flawed, which did not produce the expected effect on the perception of wastewater treatment, part of the company's concerns for environmental quality and social responsibility. As found in the longitudinal analysis at the level of respondents in branches, the percentage of responses varies around $21.00 \%$ in the two moments corresponding to 2016 and 2019 surveys (Figure 1).

In 2009 , more than $60 \%$ of those surveyed in Timisoara expressed their consent for the payment of wastewater transportation and treatment services (Figure 2); the percentage increased to $74.80 \%$ in 2019 . In this case, the trend curve shows the mood and awareness of customers to company's actions on environmental quality and social responsibility actions, due to the positive impact of organizational communication efforts has been noted. On the other hand, about half of the respondents thought it is right to pay for wastewater treatment (Figure 2), but the trend curve is decreasing in this case, thus noticing the limiting effect of organizational communication efforts directed towards a positive perception of supporting environmental quality activities and social responsibility actions. 


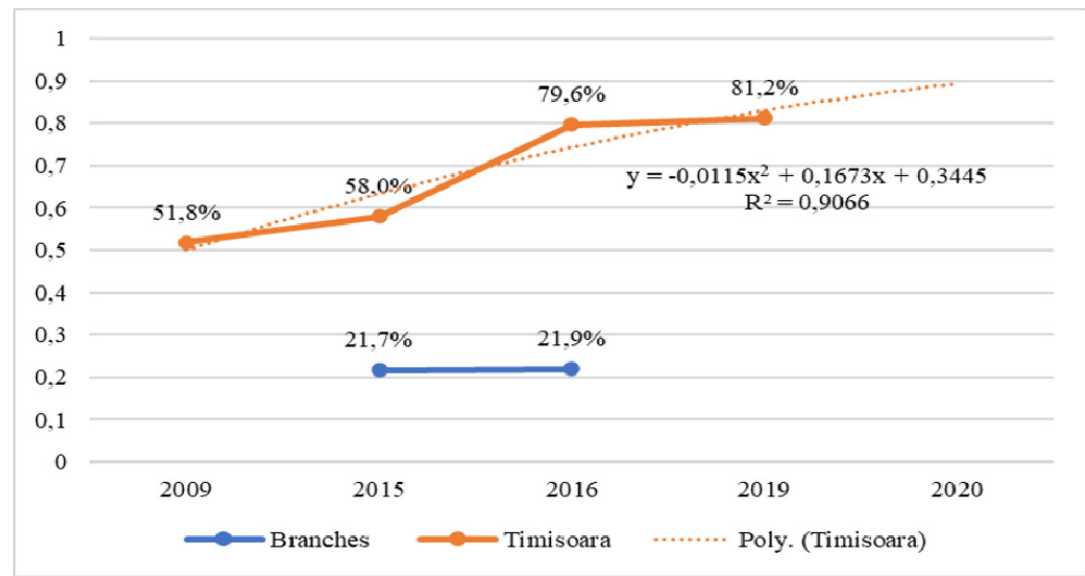

Fig. 1. Answers distribution about "The wastewater that reaches the sewerage network is treated?".

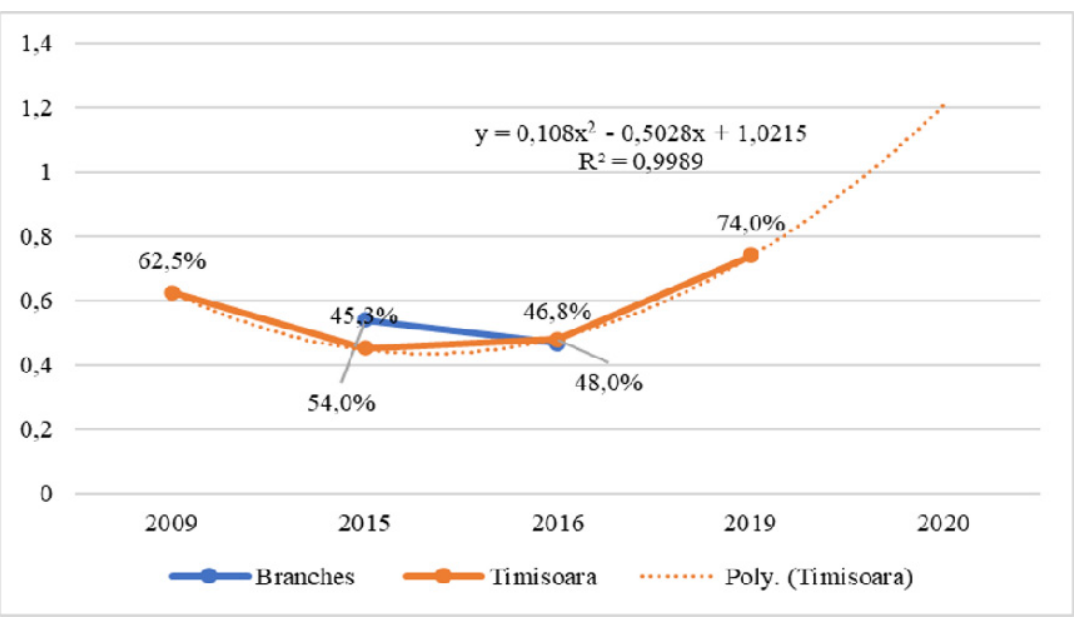

Fig. 2. Answers distribution of affirmative responses to consumers consent for payment of wastewater treatment.

\subsection{Dimension: Customers' Satisfaction}

The analysis of the "Customers' Satisfaction" dimension identified as a new aspects for improving customers' perception on social responsibility. The dimension was described by two variables according to (2).

\section{Customers' Satisfaction $=f($ service satisfaction + requests $/$ complaints solving + interaction with company employees)

The research conducted from 2009 to 2019 showed average grades above 7 for customers' satisfaction with the services offered by the company [5]. Greater notes were provided by Timisoara respondents (customers) than those in branches (Figure 3). Consequently, at the level of general satisfaction, the trend curve is slightly decreasing for Timisoara customers, compared to those in branches that is growing slightly. Explanations of these phenomena can be associated with the following findings:

- Timisoara Customers have been subject to investment pressure, associated with deterioration of the water supply process, frequent interruptions, which has led to 
increased dissatisfaction. Although the company's communication process with this category of customers was good, efficient, they did not understand the company's efforts and showed no understanding of it;

- Respondents/customers from the branches were more severe in appreciating satisfaction with the services offered, due to the lack of knowledge of the operating mode, of the investments processes (because of inadequate communication), and the weak collaboration of the water company with the local authorities affiliated to the branches (a solution for raising the level of information and creating a positive perception of the services offered).

In Figure 4 is presented the customers' perception on how the water company has solve their claims, problems, or requests. In 2009, the respondents in Timisoara gave an average mark of 5.97 as how the problem complained of was solved, in 2015 this average score rises to 6.37. In 2016, the respondents belonging to the Aquatim subsidiaries (branches) awarded an average mark of 5.04, while those from Timisoara appreciated the way of solving complaints with an average mark of 9.65. In 2019, respondents from subsidiaries gave an average score of 7.24, and those in Timisoara, an average score of 9.16 (Figure 4). As can be seen, the trend curves of the two categories of respondents show an increased level of satisfaction with the way the company intervened in the solution of the problems (with major differences of perception between the clients in Timisoara and those in subsidiaries).

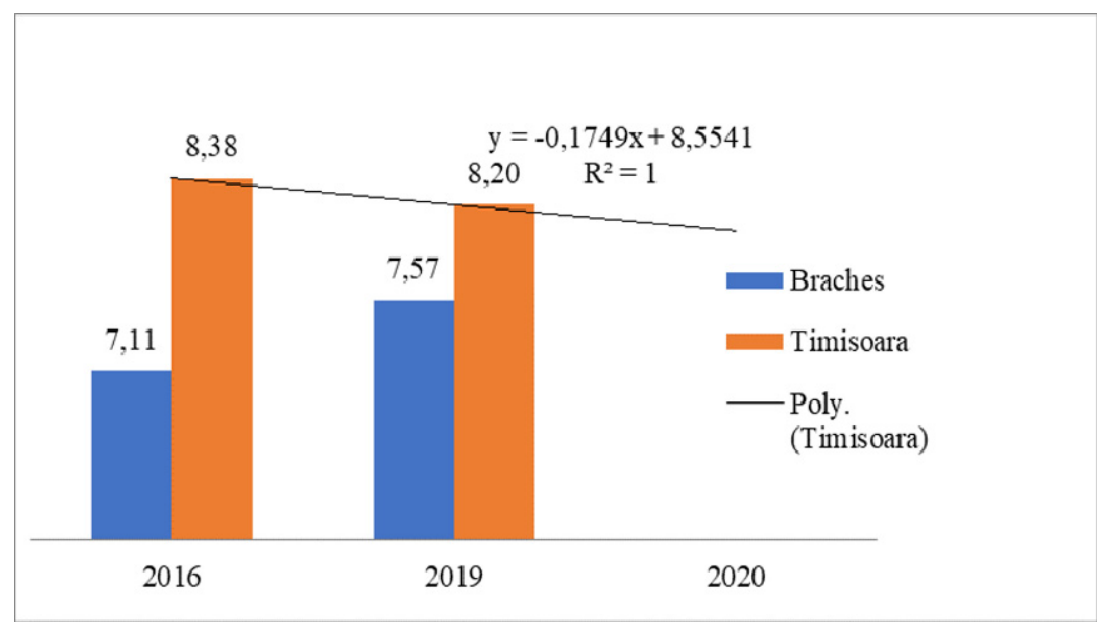

Fig. 3. Grades given for the satisfaction on the services provided by Aquatim company.

However, from the results shown in Figure 4, the responses percept an increased operational capacity of the company in crisis management, starting from good communication with consumers, as well as appreciation of the quality of intervention works appreciated with good grades. Thus, in 2019, the respondents/customers in the branches appreciated the average improvement of the drinking water system by 7.24 , while the respondents in Timisoara appreciated this activity with a score of 9.16 (score, grades from 1 to 10$)$. 


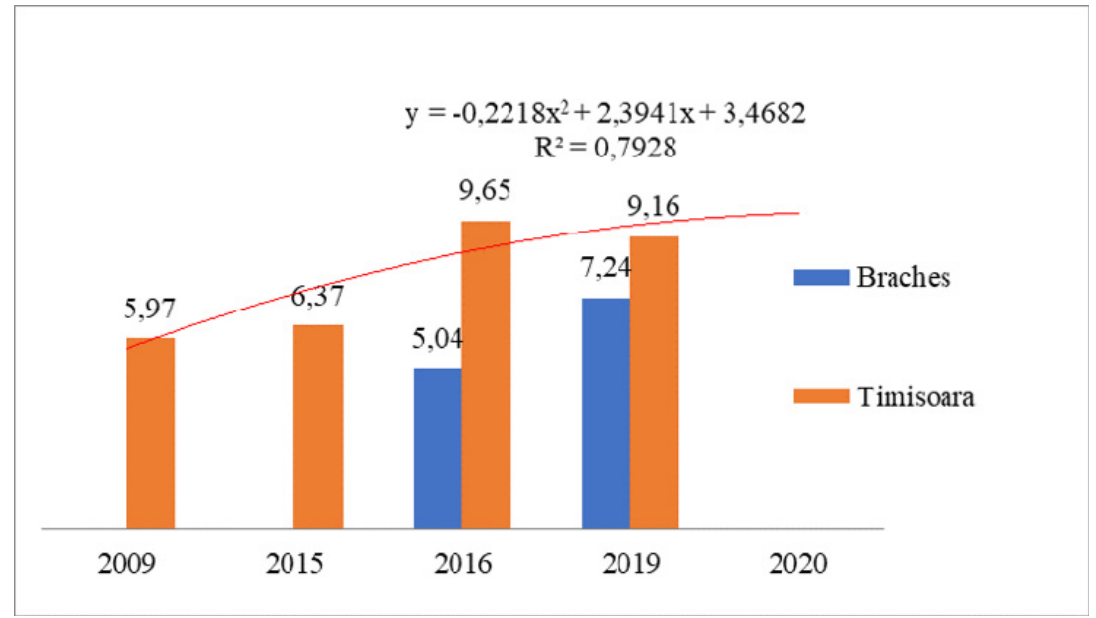

Fig. 4. Grades given for the way the company solve the water distribution problems.

Finally, some aspects that define the interaction of customers, consumers with the staff of Aquatim company were analysed.

As can be seen from Figure 5, in 2009, 20.5\% of household customers in Timisoara met or encountered employees of Aquatim SA Timisoara. Their number increased steadily by 2016 (to $35 \%$ ), and then slightly decreased until 2019 (only $29.4 \%$ of respondents admitted they had met or encountered company staff). The same slight decrease from 2016 to 2019 is also observed for branch customers (from $36.4 \%$ in 2016 to 30\% in 2019). The downward trend of the two respondents / clients shows an increase in the company's operating capacity (due to the technical optimization of the water distribution network through investments), while increasing the customer's trust towards the company.

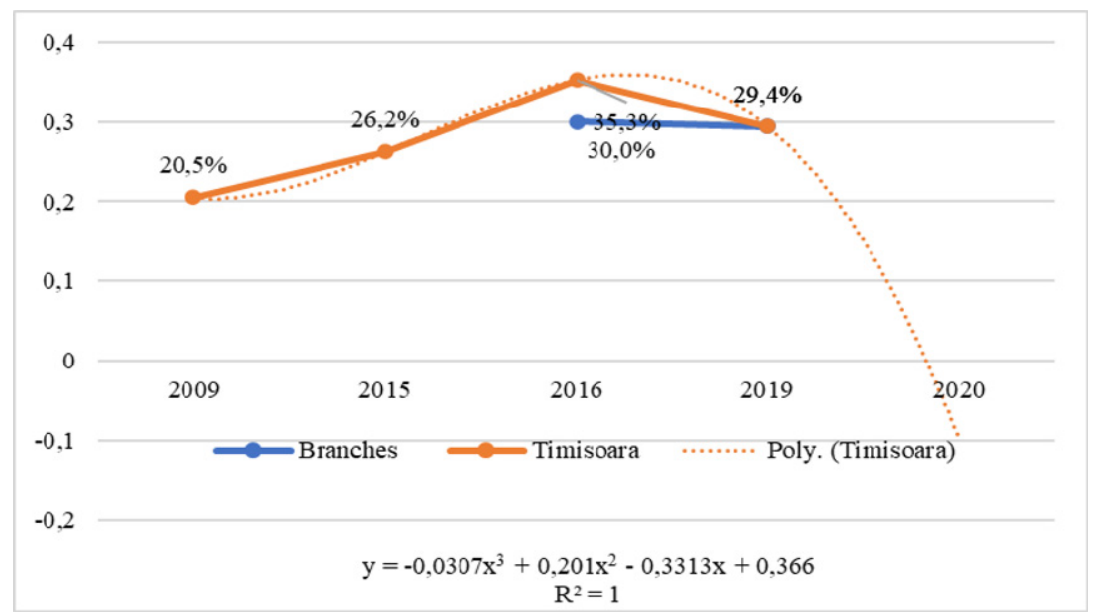

Fig. 5. Percentage of people who met, encountered Aquatim employees.

The behaviour of the water company employees was characterized by the appreciations, answers given by the respondents (notes given) on three aspects: "promptness", "respect" and "efficiency". The grades for "promptness" of the employees (Figure 6), there is an increasing trend of positive perception for Timisoara customers, from $8.19 \%$ in 2009 to 9.31 in 2019. At branches level, the perception of employees' "promptness" remains at a constant value of $8.4 \%$ for both surveys in 2016 and 2019. Regarding the grades for 
"respect" of the employees towards the customers in Timisoara, they gave an average mark of $8.87 \%$, during the analysis period, the dynamics being an intense one (Figure 7): (a) 2009 - 2015, customer perceptions are down from 8.59\% to 7.87\%; (b) In 2015 - 2019, customer perceptions are rising to $9.52 \%$.

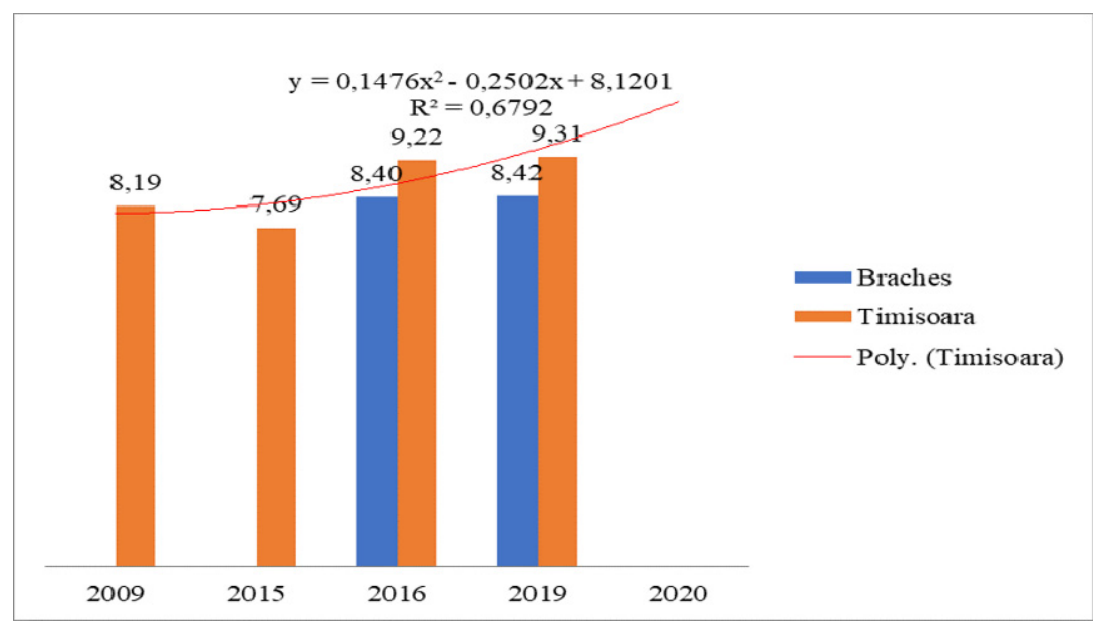

Fig. 6. Grades given for company employees on "promptness".

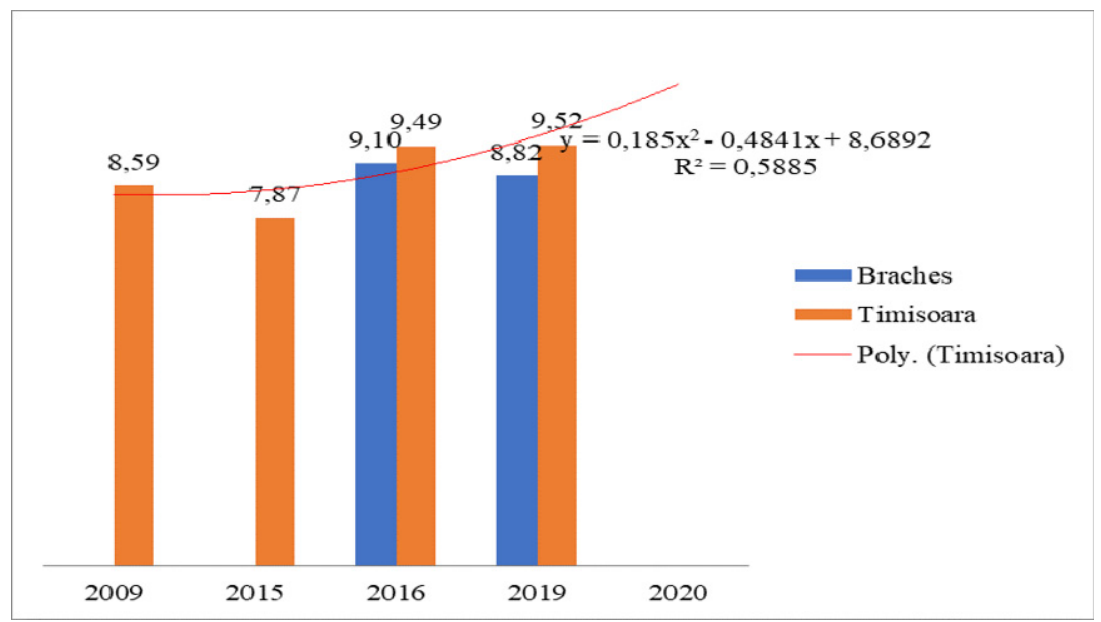

Fig. 7. Grades given for company employees on "respect".

The grades trend of the respondents in branches is decreasing from 9.10 in 2012 to 8.82 in 2015 (Figure 8). As for the other indicators of customer relationship characterization with Aquatim employees, and in the case of "efficiency", there is an increasing trend, in the period 2005 - 2015, for the respondents from Timisoara. As for the respondents in the branches, the grades expressed for the employees' "efficiency" are positioned at about one unit of 10 down and are on a declining trend between 2012 and 2015 (Figure 8). 


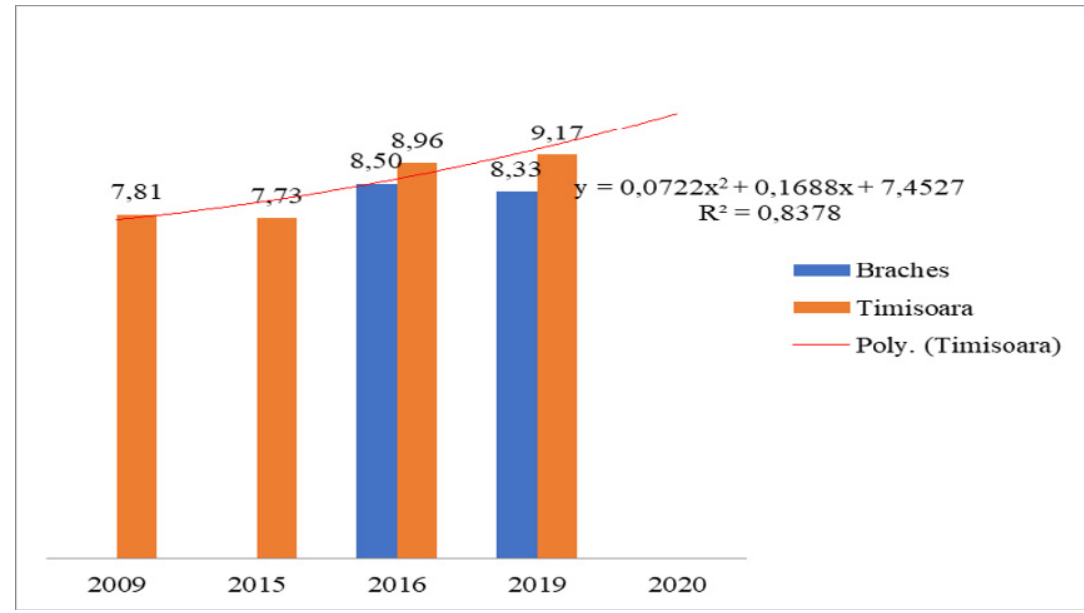

Fig. 8. Grades given for company employees on "efficiency".

\section{Conclusions and final remarks on the research results}

The investigation findings about "Ecological Awareness" dimension revealed major differences in the communication process between the Timisoara and the branch groups. As noted, the company has deficiencies in the tactical level of the communication process in the case of customers from branches and this can be associated with an inadequate and inefficient effort that impacts a poor perception of the need for wastewater treatment and assumption of payment for these services, by customers. Thus, it is imperative that the communication process to be improved, redefined to eliminate these differences in the future.

The direction of changes in the communication process is also suggested by the analysis of the social responsibility dimensions associated with the "Ecological Awareness" dimension:

- The social dimension, associated with the customers right to water and a clean environment, is well perceived by the Timisoara customers (increasing trend for the period $2009-2020$ ). The customers/respondents in the branches can be associated only $20 \%$ of them with similar issues;

- The economic dimension, related to the acceptance for the payment of wastewater treatment services, has a positive trend towards publicity in Timisoara and a declining trend for household customers in branches;

- The ecological and cultural dimensions were perceived between the group of respondents in Timisoara and the one in the branches, the first one being better positioned, interested in the ecological aspects, then the second one.

The conclusions of the research on the characterization of the "Customers' Satisfaction" dimension revealed major differences in the customers' perception because of the communication process regarding the company activity, between the group of customers (Timisoara and the branches). The research has revealed deficiencies at the tactical level of the communication process in the case of branches which can be interpreted with the inability of Aquatim company's specialized personnel to implement a suitable and differentiated message for this category of customers, as well as the lack of involvement of local municipalities in the communication process. Thus, research has highlighted the need to redefine organizational communication. 
The analysis of the dimensions of social responsibility associated with the "Customers' Satisfaction" dimension allowed the following conclusions to be drawn:

- The social dimension is associated with the respondents' perception on how the company solve their problems by ensuring continuity access to the drinking water source;

- The ethical dimension was confirmed by the efficient, responsible, and respectful behaviour of the company's employees towards the customers;

- The economic dimension is partly evidenced by the promptness of interventions in crisis management;

- Value system dimension - the analysis highlighted the manifestation of the following values of Aquatim company: integrity, loyalty, accountability, transparency, ethics, and professionalism proven by the notes of customers to the qualities of the company's employees and to the general behaviour in crisis situations requests, complaints, remedies);

- The cultural dimension is partially satisfied due to the differences in the perception of the customers in the branches compared to those in Timisoara relative to the services offered by the company.

Based on the statistical data analysis of the longitudinal analysis, it has been represented the theoretical model relative to the customers' perceptions on social responsibility in the case of Aquatim water company and for the available data from 2005 to 2015 (Figure 9, showing the regression function standard coefficient because all relations are statistical significance, significance level values are below 0.05).

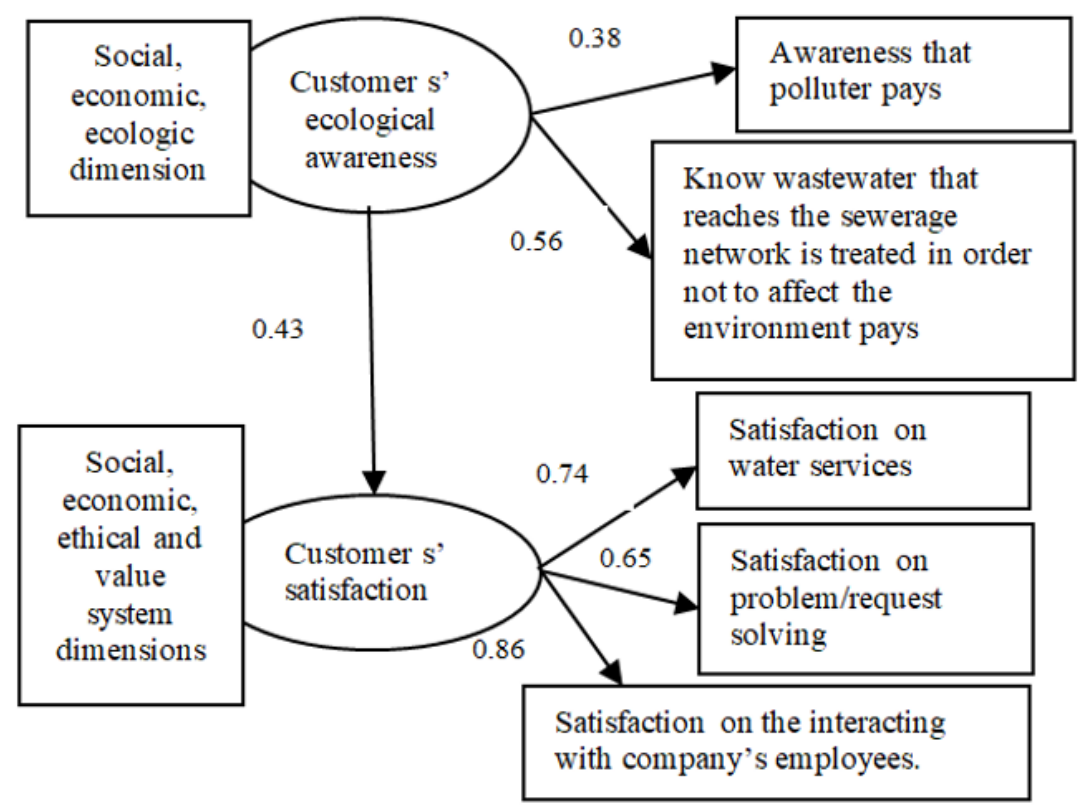

Fig. 9. The theoretical model associated to the longitudinal study.

The authors are grateful to Aquatim company in Timisoara, Romania (https://www.aquatim.ro/) for providing the initial databases of 2005-2015 surveys and, without which, this transversal analysis would not have been possible.

The longitudinal study was undertaken through the AUF-RO Partnerships and was funded from the Program PN III/3.1, grant no. 06-AUF /01.03.2019. 


\section{References}

1. D. J. Hand, Practical Longitudinal Data Analysis. Routledge, (2017)

2. H. Ahsan, Y. Chen, F. Parvez, L. Zablotska, M. Argos, I. Hussain, Am. J. of Epidem. 163 (12), 1138-1148 (2006)

3. M. Lee, B. Tansel, M. Balbin, Resources, Conservation and Recycling 56 (1), 1-6, (2011)

4. T. Stukel, E. Greenberg, B. Dain, F. Reed, N. Jacobs, Env. sc. \& tech. 24 (4), 571-575, (1990)

5. P. Bansal, Strategic Manag. J. 26 (3), 197-218, (2006)

6. C. Borca, The Role Corporate Communication in the Perception of Social Responsibility's Dimensions, Ph D. Thesis, Politehnica Publishing House, Timisoara, (2018) 